Network Working Group

Request for Comments: 5105

Category: Standards Track
O. Lendl

enum.at

December 2007

\title{
ENUM Validation Token Format Definition
}

Status of This Memo

This document specifies an Internet standards track protocol for the Internet community, and requests discussion and suggestions for improvements. Please refer to the current edition of the "Internet Official Protocol Standards" (STD 1) for the standardization state and status of this protocol. Distribution of this memo is unlimited.

Abstract

An ENUM domain name is tightly coupled with the underlying E.164 number. The process of verifying whether the Registrant of an ENUM domain name is identical to the Assignee of the corresponding E.164 number is commonly called "validation". This document describes a signed XML data format -- the Validation Token -- with which Validation Entities can convey successful completion of a validation procedure in a secure fashion.

Table of Contents

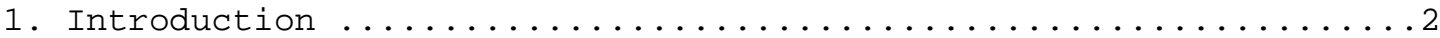

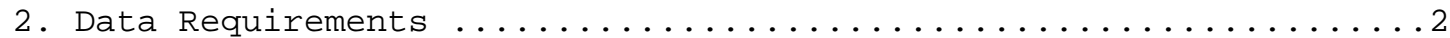

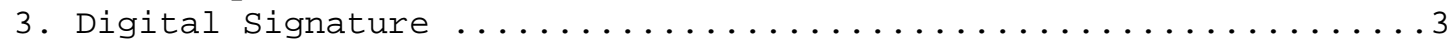

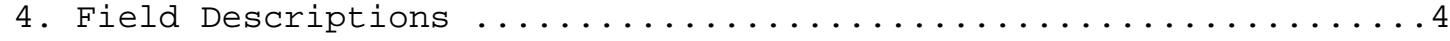

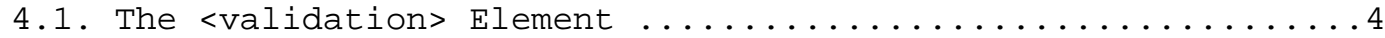

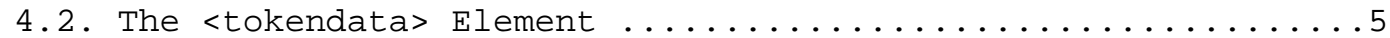

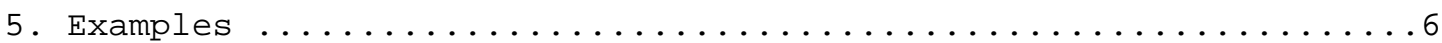

5.1. Unsigned Token without Registrant Information ........6

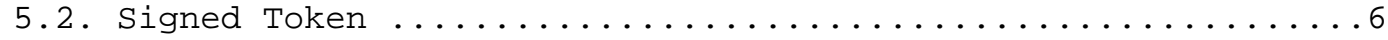

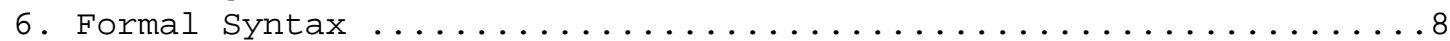

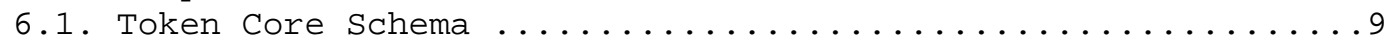

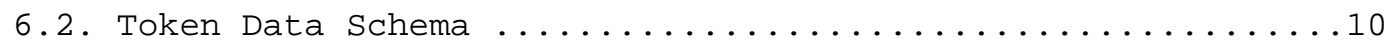

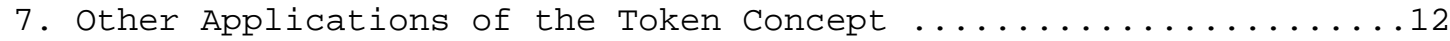

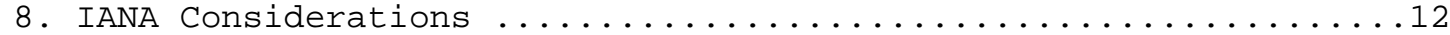

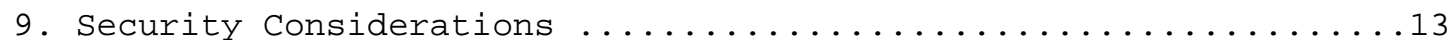

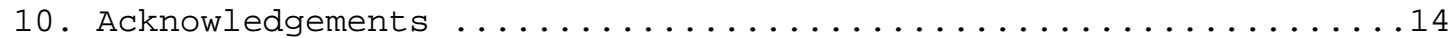

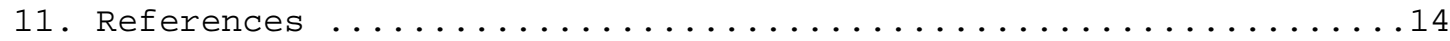

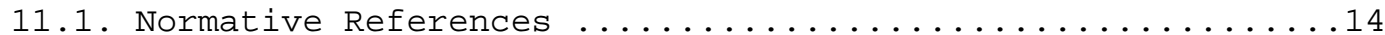

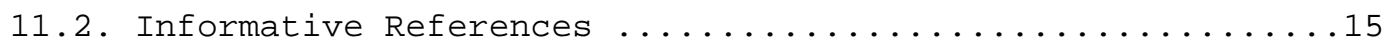




\section{Introduction}

In the case where an ENUM (E.164 Number Mapping [1]) domain name corresponds to an existing E.164 number [2], the delegation of this domain needs to be authorized by the Assignee of the corresponding E.164 number. In the role model described in [15], the entity that performs this check is called the Validation Entity (VE).

By conveying an ENUM Validation Token -- a signed XML document -- to the Registry, a VE certifies that delegation requirements have been met and are current.

The key words "MUST", "MUST NOT", "REQUIRED", "SHALL", "SHALL NOT", "SHOULD", "SHOULD NOT", "RECOMMENDED", "MAY", and "OPTIONAL" in this document are to be interpreted as described in RFC 2119 [3] .

\section{Data Requirements}

In this model, the Token is the only piece of data passed from the VE to the Registry. Therefore, the Token needs to contain at least as much information as the Registry requires to grant the delegation of the requested ENUM domain according to its registration policy. As such, the Registry will need confirmation that:

o the Token was created by an accredited VE,

o the Token's duration of validity conforms to the policy,

- the validation procedure employed has met minimum requirements as set forth by policy,

o and that the Token is protected against tampering and replay attacks.

Beyond such mandatory information, the Token may optionally include number holder information, in particular, to simplify future revalidations.

For example, if initial validation requires the steps "Check the identity of the Registrant" and "Check the ownership of an E.164 number", then a later revalidation only needs to re-check the ownership as the identity of the Registrant does not change.

As the Token will be included (see e.g., [16]) in XML-based Registry/ Registrar protocols like the Extensible Provisioning Protocol (EPP) [13], it is a natural choice to use XML to encode Validation Tokens. 


\section{Digital Signature}

According to the architecture model the propriety of an ENUM delegation depends on the trust relationship between the Registry and the VE. In general, an untrusted link between the Registry and VE should be assumed (for instance, the Token is passed along with the registration request by a Registrar, who might have no role in asserting the right-to-use). Therefore, the Token must be protected against forgery, tampering, and replay-attacks.

A digital signature on the token:

- asserts that the token was indeed generated by the indicated VE (authenticity).

- guarantees that the token was not tampered with in transit (integrity).

o enables auditing the validation process (non-repudiation).

The cryptographic signature on the token follows RFC 3275 (XML-DSIG [4]). As tokens might be transmitted as part of an already XML based protocol, the exclusive XML canonicalization [9] MUST be used. This transform guarantees that namespace declarations inherited from the surrounding $\mathrm{XML}$ do not invalidate the signature. In order to make the signature an integral part of the token, the "enveloped"-signature mode is employed. The signature covers all information contained in the Token.

XML-DSIG offers a number of cryptographic algorithms for digesting and signing documents and recommends SHA1/RSA-SHA1. Recent advances in cryptanalysis have cast doubt on the security of SHA1, thus rendering this recommendation obsolete (see e.g., the Security Considerations of [14]). RFC 4051 [5] defines how additional algorithms can be used with XML-DSIG.

Validation Entities MUST be able to sign tokens according to XML-DSIG, MUST support RSA-SHA1 and RSA-SHA256 [5], MUST support RSA key sizes of 1024 and 2048 bits, and MUST be able to embed X.509 [10] certificates. The Registry MUST define which signature algorithms and key sizes it will accept in Validation Tokens as part of its local policy.

The choice of a RSA-based signature does not require a public key infrastructure. Whether the Registry acts as a certification authority, accepts certs from a public certification authority, or only accepts pre-registered keys is a local policy choice. 


\section{Field Descriptions}

The Validation Token is structured into three parts: the basic validation information, additional information about the Registrant, and the digital signature. The XML schema can be found in section 6 .

4.1. The <validation> Element

A token MUST contain a <validation> element that contains the following:

- A single validation "serial" attribute identifying a validation token for a certain VE. It must be unique per VE.

- A single <E164Number> element containing the underlying E.164 number in fully qualified (international) format.

- An optional <laste164Number> element. If present, it indicates that the whole number block starting with $\langle E 164$ Number $>$ up to and including <lastE164Number> has been validated. To avoid ambiguity, both numbers MUST be of the same length.

o A single <validationEntityID> element identifying the VE.

- A single <registrarID> element identifying the Registrar on whose behalf the validation was performed.

- A single <methodID> element identifying the method used by the VE for validation.

- A single <executionDate> attribute containing the date of validation formatted as "full-date" according to RFC 3339 [6].

- An optional <expirationDate> attribute marking the expiration date of the validation token formatted as "full-date" according to RFC 3339. The Registry will automatically revoke the delegation at this date unless a new Token has been submitted that extends the lifetime of the validation. A missing <expirationDate> indicates infinite validity of the Token.

The format and the uniqueness-constraints of these IDs is left to the local policy of the Registry. 
4.2. The <tokendata> Element

A token may contain a <tokendata> section containing information about the number holder, consisting of the following elements:

- A single corganization> element containing the full name of the organization to which the Registrant is affiliated.

- A single <commercialregisternumber> element. If the Registrant is a company, then this field can be used to uniquely identify this company by its official registration number within the local country. The interpretation of this field is thus country-specific.

- A single <title> element.

- A single <firstname> element.

- A single <lastname> element.

- A single <address> section containing the following elements:

* A single optional <streetName>

* A single optional <housenumber>

* A single optional <postalCode>

* A single optional <locality>

* A single optional <countystateorProvince>

* A single optional <ISOcountryCode>

- Up to 10 <phone> elements containing full E.164 numbers.

- Up to 10 <fax> elements containing full E.164 numbers.

o Up to 10 <email> elements.

All elements directly under <tokendata> are optional. The <ISOcountryCode> element specifies the country using the alpha-2 country code from ISO 3166-1:2006 [11] (including updates published by the 3166 Maintenance Agency). The definition of the first five elements within the <address element conforms to the second version of the E.115 Computerized Directory Assistance [17]. 


\section{Examples}

5.1. Unsigned Token without Registrant Information

This basic Token without any information about the Registrant and without the cryptographic signature shows the basic layout of the Token.

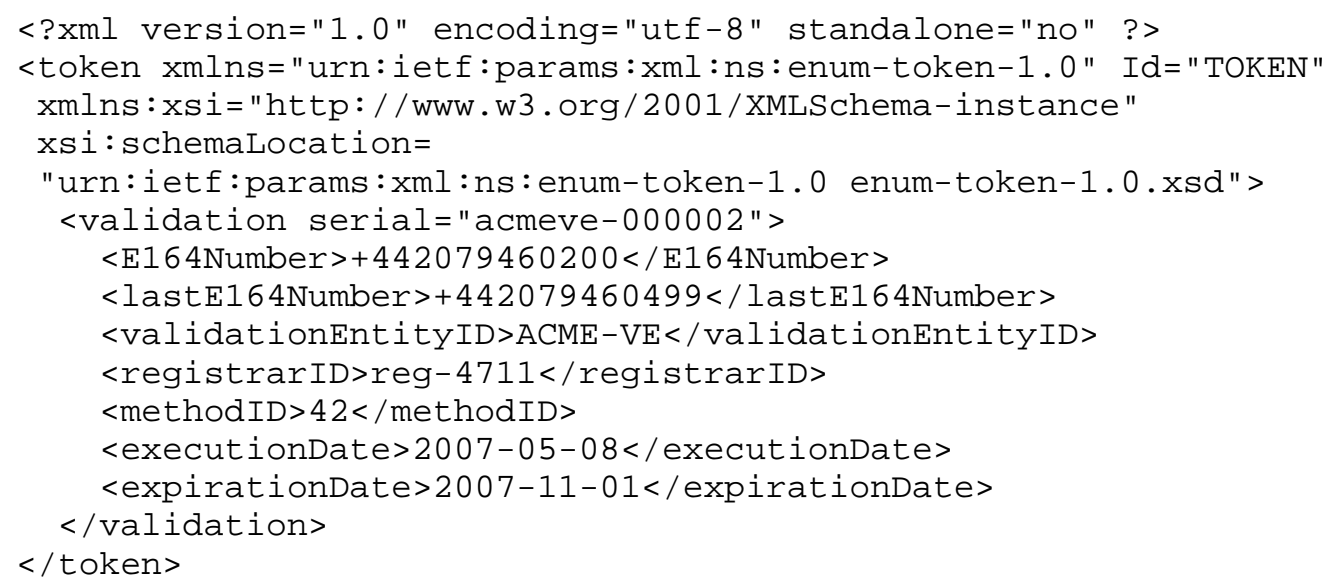

\subsection{Signed Token}

This example uses an X.509 based signature that includes the certificate of the signing validation entity. Thus, the validity of the signature can be verified without the need for a key-server. A valid signature is a necessary, but not sufficient, condition for a valid Token. Any entity evaluating a Token needs to check other factors as well, e.g., the certificate and the XML schema.

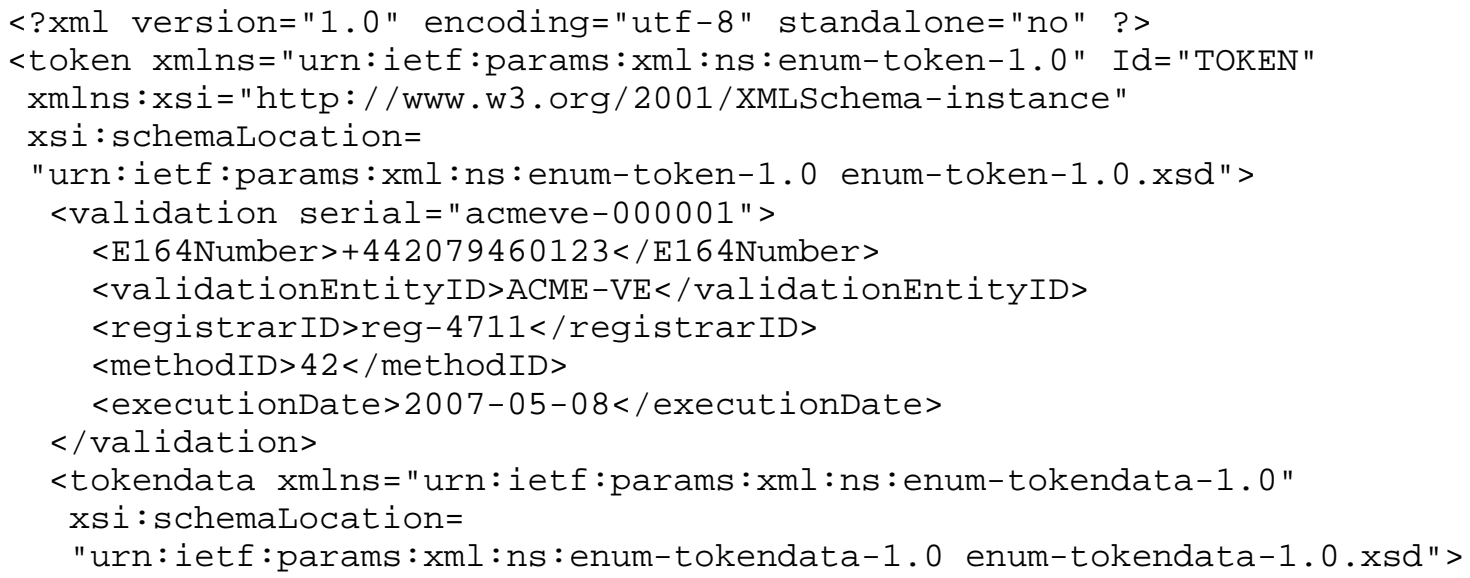




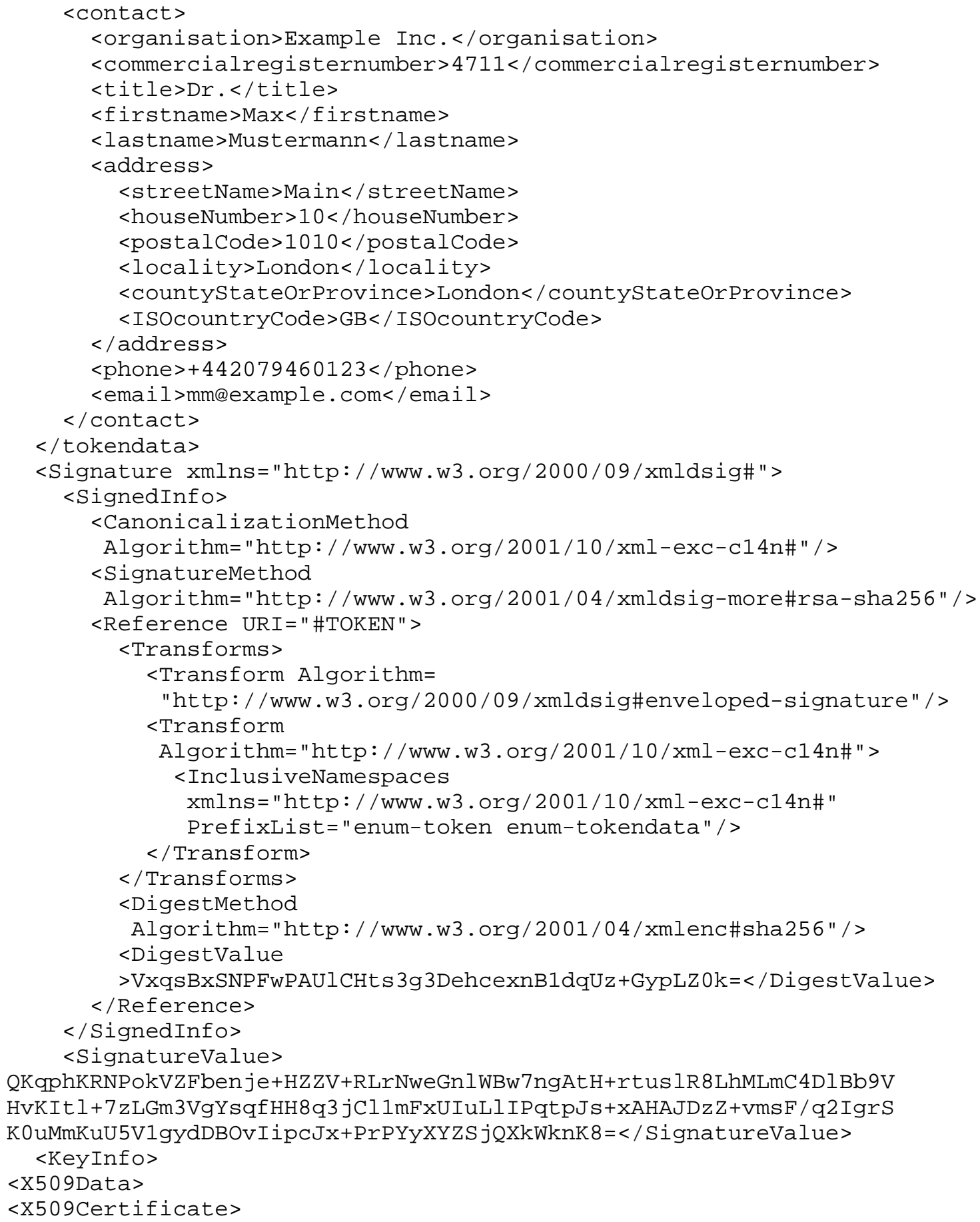


MIIDZjCCAs+gAwIBAgIBBDANBgkqhki G9w0BAQQFADBOMQswCQYDVQQGEwJBVDEP MA0GA1 UEBXMGVml l.bm5hMRQwEgYDVQQKEwtCTOZI IENI CnRzLjEbMBkGA1UEAxMS QOVSVFMuYm9maC5wcml2 LmF OMSEwHwYJKoZI hvcNAQkBFhJ jZXJ0c0Bib2ZoLnBy aXYuYXQwHh CNMDQwNz I wMTMxNTA 5Wh CNMDUwNz I wMTMxNTA5W jB/MQswCQYDVQQG EWJBVDEKMAgGA1UECBMBLTEPMA0GA1 UEBXMGVml lbm5hMR0wGwYDVQQKEXRBY21l IEVOVU0 gVmF s aWRhdGI vb jEQMA 4 GA1 UEAxMHYWNt ZS 1WRTE IMCAGCS GGS Ib3DQEJ ARYTbm9ib2R5QGVudW0tYWNtZS5hdDCBnzANBgkqhkiG9w0BAQEFAAOB jQAwgYkC gYEArJP C jMF c 54 / zwztSdQXGxUtodJT9r1qGI2 1QPN jLvtP Jg93+7o5SIOsZGSpg zWb ztDAV5qc7P HzWUV Iy f 6MbM5 qSgQDVr jNRhTosNtyqmwi23BH52SKkX3P 7eGit LmqEkiUZRxZhZ6upRbt cqvKSwmXitvW4 zXZhkVHYJZ2 HuMCCAwEAAaOB / DCB+TAJ BgNVHRMEA jAAMCWGCWCGSAGG+EIBDQQfFh1P CGVUU1NMIEdlbmVYYXRIZCBDZXJ0 aWZpY2F0ZTAdBgNVHQ4 EF gQUyK4 ot TQtvv6KdSIMBOP T5Ve18 JgwgZ4GA1UdIwSB 1 jCBk 4 AUvfPadpm0 HhmZx2iAVumQTwgnG2eheKR2MHQxCzAJBgNVBAYTAkFUMQ8w DQYDVQQHEWZWaWVubmEXFDASBgNVBAOTCO JPRkggQ2VydHMuMRsWGQYDVQQDEXJD RVJUUy5ib2ZoLnByaXYuYXQxITAfBgkqhkiG9w0BCQEWEmNl cnRzQGJvZmgucHJp di5hdI IBADANBgkqhki G9w0BAQQFAAOBgQCB 9CHBnIUhrdic4h5Ar4hdx jHSQkDH s JWd+MYrNcuSrv3TIOsUkUgNpNNhmkZPtiXqfy3388 IRdJt Ji LWXSOb/XlZHOM9 I MvwKYwhcpQ9UdM/w7VpXeqf+CEj0XSyqxGw65UsHIOijgiG/WyhSj+Lzriw7CTge P2iAJkJVC 4t $2 \mathrm{XA}==$

$</$ X509Certificate>

$</$ X509Data $>$

$</$ KeyInfo $>$

$</$ Signature $>$

$</$ token $>$

6. Formal Syntax

The formal syntax of the validation token is specified using XML schema notation [7] [8]. Two schemas are defined: The "token core schema" contains mandatory attribute definitions, and the "token data schema" defines the format of the optional "tokendata" section. The BEGIN and END tags are not part of the schema; they are used to note the beginning and ending of the schema for URI registration purposes. 


\subsection{Token Core Schema}

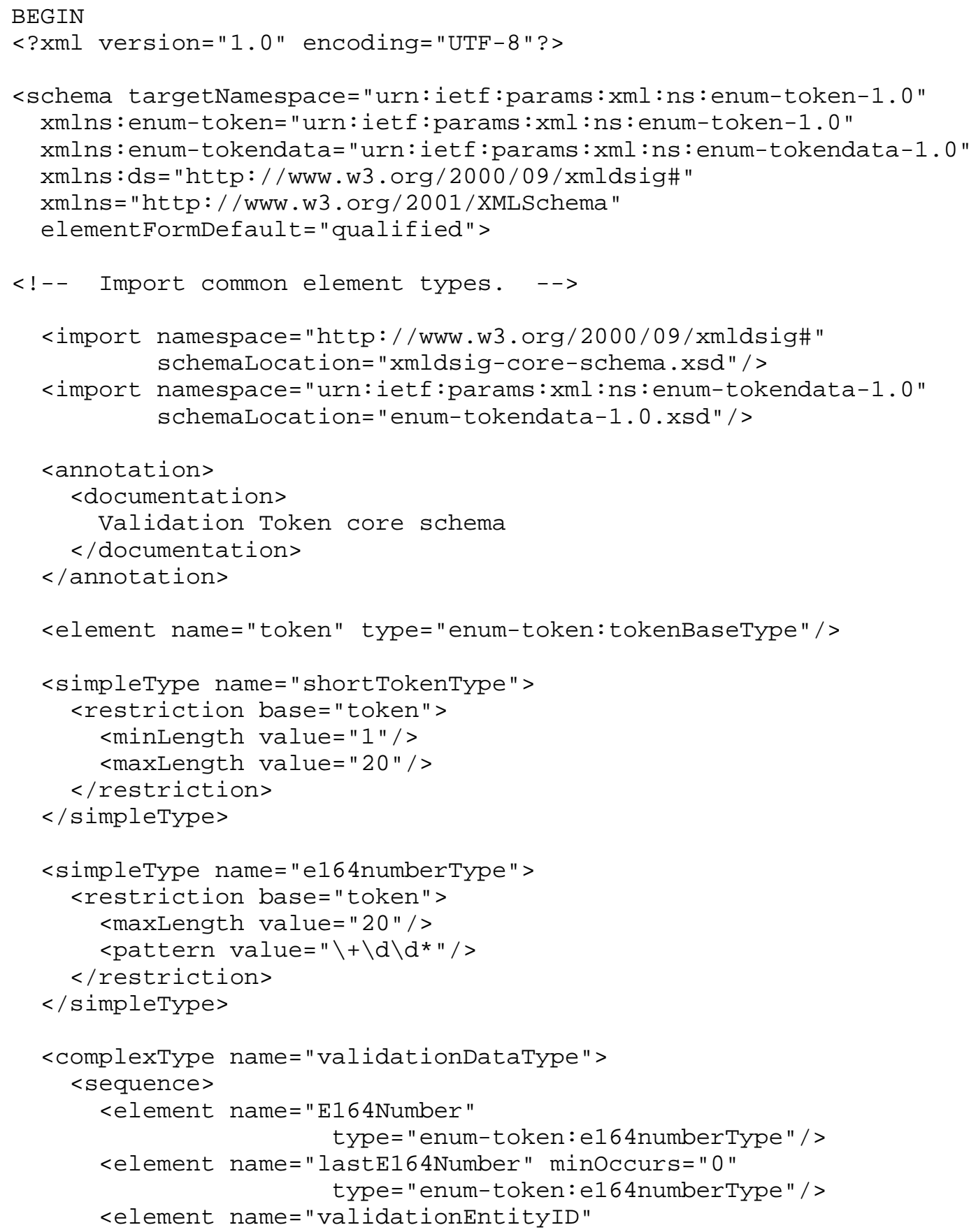




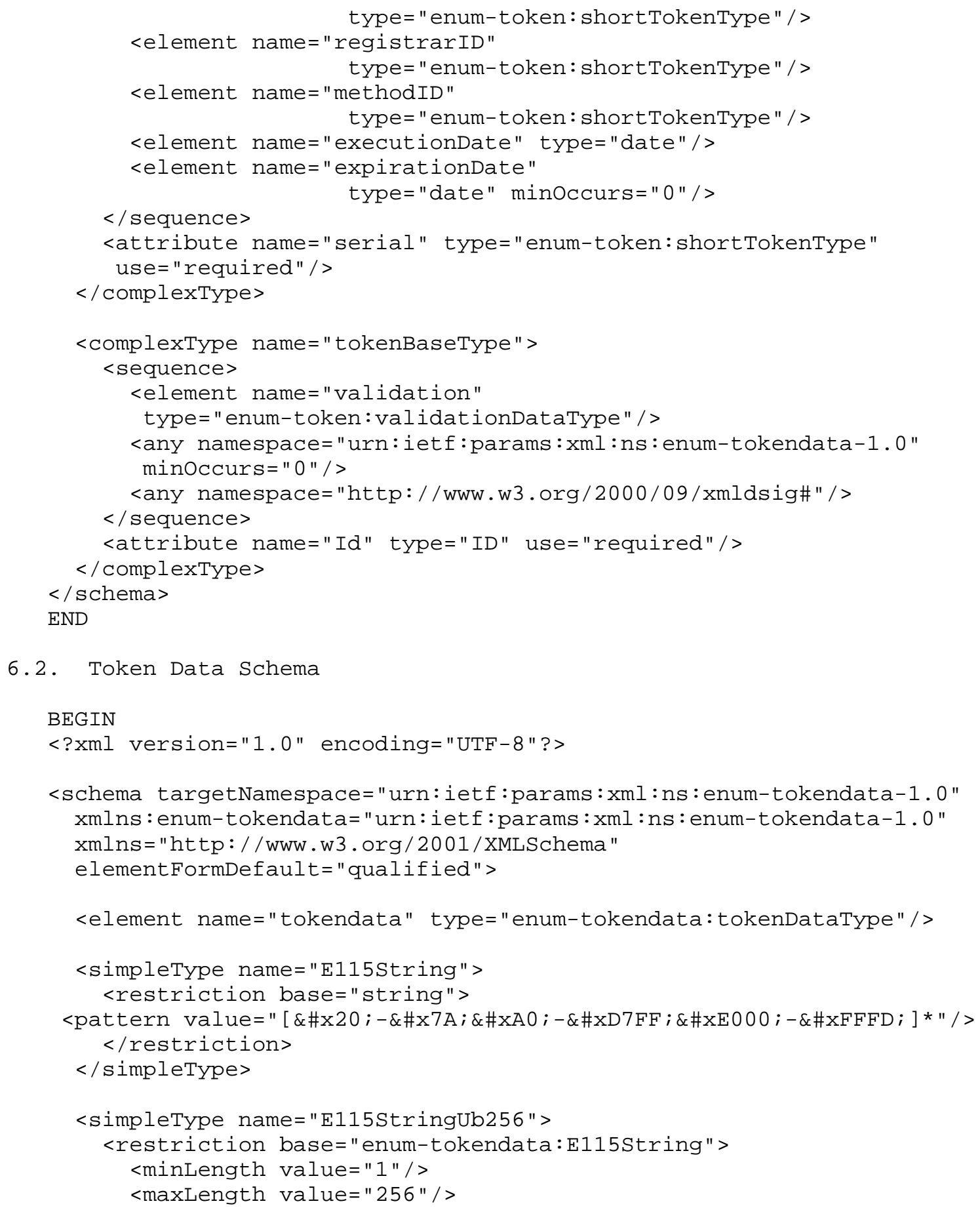




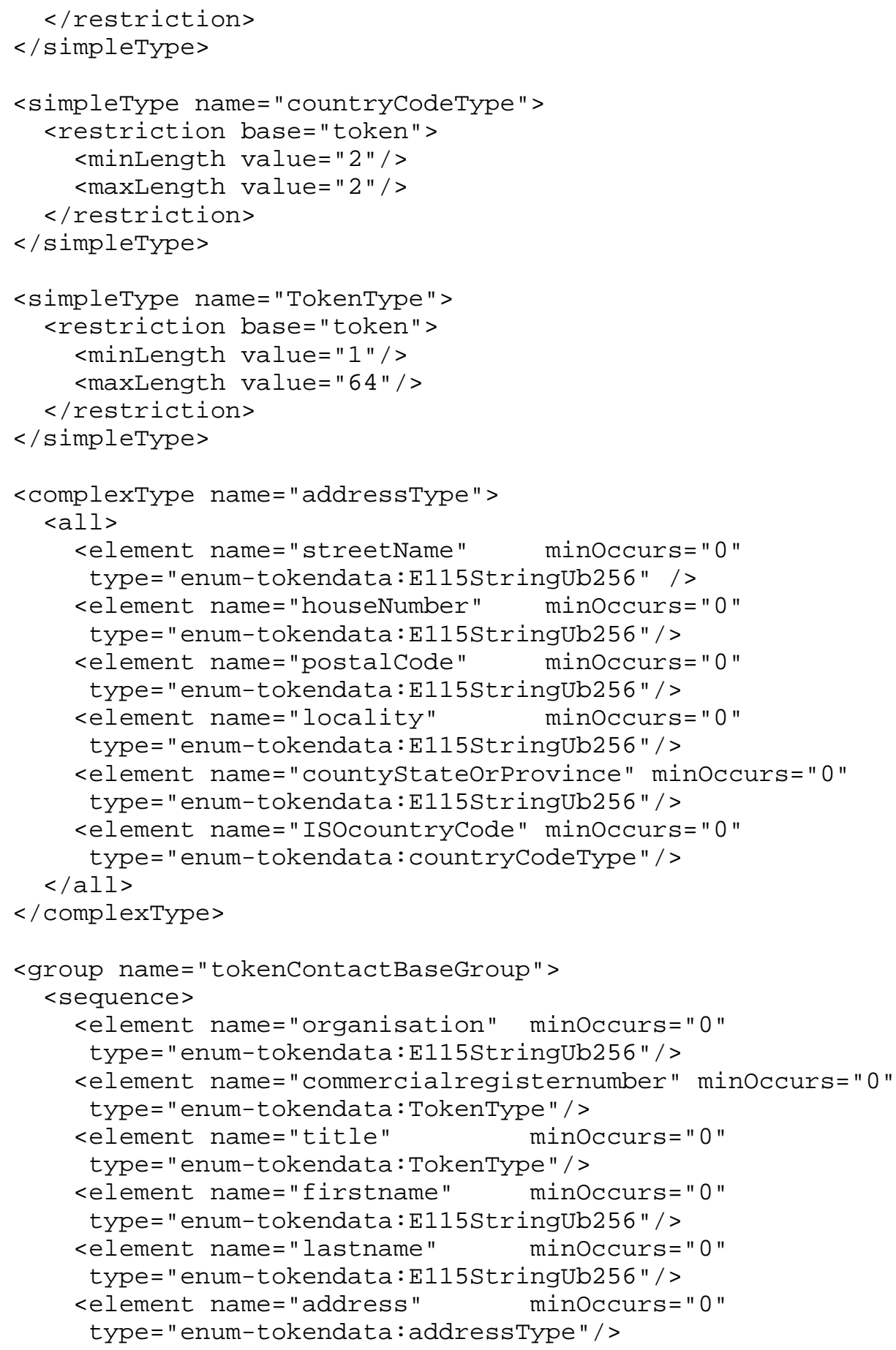




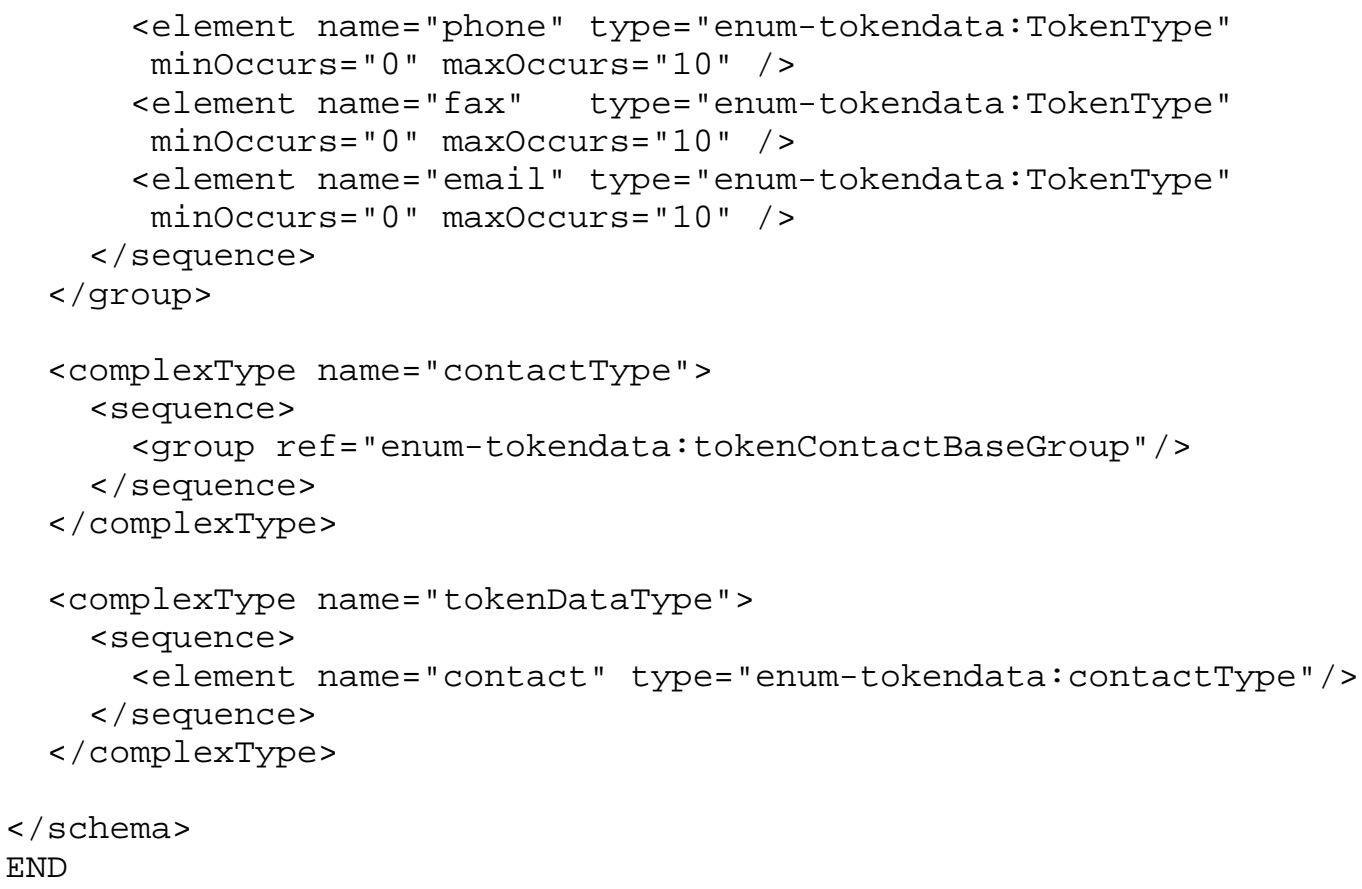

8. IANA Considerations

This document uses Uniform Resource Names (URNs) to describe XML namespaces and XML schemas conforming to a registry mechanism described in RFC 3688 [12]. IANA has made the following four URI assignments. 
1. Registration for the Token namespace:

* URI: urn:ietf:params: xml:ns:enum-token-1.0

* Registrant Contact: See the "Author's Address" section of this document.

* XML: None. Namespace URIs do not represent an XML specification.

2. Registration for the Token XML schema:

* URI: urn:ietf:params:xml:schema:enum-token-1.0

* Registrant Contact: See the "Author's Address" section of this document.

* XML: See section 6.1 of this document.

3. Registration for the Token Data namespace:

* URI: urn:ietf:params:xml:ns:enum-tokendata-1.0

* Registrant Contact: See the "Author's Address" section of this document.

* XML: None. Namespace URIs do not represent an XML specification.

4. Registration for the Token Data XML schema:

* URI: urn:ietf:params:xml:schema:enum-tokendata-1.0

* Registrant Contact: See the "Author's Address" section of this document.

* XML: See section 6.2 of this document.

The IDs used in the validationEntityID, RegistrarID, and methodID elements are subject to local policy and thus do not require IANA registration.

9. Security Considerations

The security of the Validation Token depends on the security of the underlying XML DSIG algorithms. As such, all the security considerations from [4] apply here as well. Two points from [4] merit repetition:

Transforms are used to select the relevant data for signing and discarding irrelevant information (e.g., pretty-printing and name-space local names).

The <Reference URI="\#TOKEN"> element and attribute combined with the Id="TOKEN" attribute in <token> specifies that the signature should cover the complete token. Moving the Id="TOKEN" attribute to e.g., the <tokendata> element would make the signature worthless. 
It is thus critical that the Registry not only checks whether the Token passes a generic XML-DSIG signature check, but also that:

1. the signature uses approved transforms and cryptographic algorithms.

2. the signature references the <token> element.

3. the key used in the signature belongs to an accredited VE.

The Token content is not encrypted. If local policy dictates that the information contained within the token should be confidential, then this has to be handled through a different mechanism.

When processing a delegation request, the Registry MUST verify that the information contained in the Token matches the delegation request. The <registrarID> element in the Token prevents a malicious second Registrar from using an eavesdropped Token to register a domain in his name. The Registry MUST verify that the <expirationDate> given (including the case of no given expiration date) conforms to the Registry's policy. To avert replay attacks, local policy MUST specify how long after <executionDate> the Token can be used to authorize a delegation.

10. Acknowledgements

The author would like to thank the following persons for their valuable suggestions and contributions: Michael Haberler, Alexander Mayrhofer, Bernie Hoeneisen, Michael Braunoeder, Staffan Hagnell, Lawrence Conroy, and Tony Rutkowski.

11. References

11.1. Normative References

[1] Faltstrom, P. and M. Mealling, "The E.164 to Uniform Resource Identifiers (URI) Dynamic Delegation Discovery System (DDDS) Application (ENUM)", RFC 3761, April 2004.

[2] ITU-T, "The international public telecommunication numbering plan", Recommendation E.164, May 1997.

[3] Bradner, S., "Key words for use in RFCs to Indicate Requirement Levels", BCP 14, RFC 2119, March 1997.

[4] Eastlake 3rd, D., Reagle, J., and D. Solo, "(Extensible Markup Language) XML-Signature Syntax and Processing", RFC 3275, March 2002 . 
[5] Eastlake 3rd, D., "Additional XML Security Uniform Resource Identifiers (URIs)", RFC 4051, April 2005.

[6] Klyne, G. and C. Newman, "Date and Time on the Internet: Timestamps", RFC 3339, July 2002 .

[7] Maloney, M., Beech, D., Mendelsohn, N., and H. Thompson, "XML Schema Part 1: Structures", W3C REC REC-xmlschema-1-20010502, May 2001 .

[8] Malhotra, A. and P. Biron, "XML Schema Part 2: Datatypes", W3C REC REC-xmlschema-2-20010502, May 2001.

[9] Eastlake, D., Boyer, J., and J. Reagle, "Exclusive XML Canonicalization Version 1.0", W3C REC REC-xml-exc-c14n20020718, July 2002 .

[10] International Telecommunications Union, "Information technology - Open systems Interconnection - The Directory: Public-key and attribute certificate frameworks", ITU-T Recommendation X.509, ISO Standard 9594-8, March 2000.

[11] International Organization for Standardization, "Codes for the representation of names of countries and their subdivisions -Part 1: Country codes, 2nd edition", ISO Standard 3166 , November 2006 .

[12] Mealling, M., "The IETF XML Registry", BCP 81, RFC 3688, January 2004 .

\subsection{Informative References}

[13] Hollenbeck, S., "Extensible Provisioning Protocol (EPP)", RFC 4930, May 2007 .

[14] Schaad, J., Kaliski, B., and R. Housley, "Additional Algorithms and Identifiers for RSA Cryptography for use in the Internet X.509 Public Key Infrastructure Certificate and Certificate Revocation List (CRL) Profile", RFC 4055, June 2005.

[15] Mayrhofer, A. and B. Hoeneisen, "ENUM Validation Architecture", RFC 4725, November 2006.

[16] Hoeneisen, B., "ENUM Validation Information Mapping for the Extensible Provisioning Protocol", RFC 5076, December 2007.

[17] ITU-T, "Computerized Directory Assistance Version 2", Recommendation E.115v2, October 2005. 


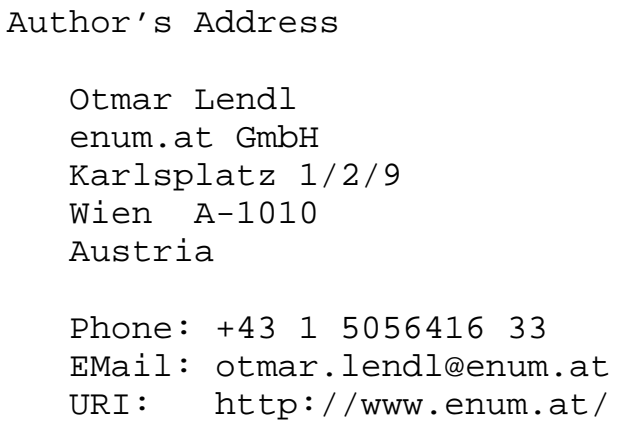


Full Copyright statement

Copyright (C) The IETF Trust (2007).

This document is subject to the rights, licenses and restrictions contained in BCP 78, and except as set forth therein, the authors retain all their rights.

This document and the information contained herein are provided on an "AS IS" basis and THE CONTRIBUTOR, THE ORGANIZATION HE/SHE REPRESENTS OR IS SPONSORED BY (IF ANY), THE INTERNET SOCIETY, THE IETF TRUST AND THE INTERNET ENGINEERING TASK FORCE DISCLAIM ALL WARRANTIES, EXPRESS OR IMPLIED, INCLUDING BUT NOT LIMITED TO ANY WARRANTY THAT THE USE OF THE INFORMATION HEREIN WILL NOT INFRINGE ANY RIGHTS OR ANY IMPLIED WARRANTIES OF MERCHANTABILITY OR FITNESS FOR A PARTICULAR PURPOSE.

Intellectual Property

The IETF takes no position regarding the validity or scope of any Intellectual Property Rights or other rights that might be claimed to pertain to the implementation or use of the technology described in this document or the extent to which any license under such rights might or might not be available; nor does it represent that it has made any independent effort to identify any such rights. Information on the procedures with respect to rights in RFC documents can be found in BCP 78 and BCP 79 .

Copies of IPR disclosures made to the IETF Secretariat and any assurances of licenses to be made available, or the result of an attempt made to obtain a general license or permission for the use of such proprietary rights by implementers or users of this specification can be obtained from the IETF on-line IPR repository at http://www.ietf.org/ipr.

The IETF invites any interested party to bring to its attention any copyrights, patents or patent applications, or other proprietary rights that may cover technology that may be required to implement this standard. Please address the information to the IETF at ietf-ipreietf.org. 